\title{
uvby photometry of the magnetic CP stars 45 Leonis, HR 4330, 49 Herculis, and HR 6718 ${ }^{\star}$
}

\author{
S.J. Adelman, K.E. Rayle, and C.-L.M. Pi \\ Department of Physics, The Citadel, 171 Moultrie Street, Charleston SC 29409, U.S.A.
}

Received December 22, 1998; accepted February 8, 1999

\begin{abstract}
Differential Strömgren uvby photometric observations from the Four College Automated Photoelectric Telescope are presented for the magnetic CP stars 45 Leo, HR 4330, 49 Her, and HR 6718. We refined Winzer's periods for 45 Leo and HR 6718 to 1.443820 days and 0.51899 days, respectively. For HR 4330 our period of 3.516 days is quite different from that of Winzer. That for $49 \mathrm{Her}$, 0.93663 days, is an alias of Winzer's.
\end{abstract}

Key words: stars: chemically peculiar — stars: individual: 45 Leo, HR 4330, 49 Her, and HR 6718

\section{Introduction}

The anomalous photospheric abundances of the magnetic Chemically Peculiar (mCP) stars, whose effective temperatures and surface gravities are similar to those of main sequence B, A, and early F stars, are thought to be produced by hydrodynamical processes including radiative diffusion and gravitational settling in radiative envelopes which have strong magnetic fields (Michaud \& Proffitt 1993 and references therein). The resulting abundance distributions depend upon the magnetic field, should be patchy, and affect the emergent flux distribution (Shore \& Adelman 1974). This is necessary to explain their photometric, magnetic, and spectrum variability. Differential photometric studies with the $0.75-\mathrm{m}$ Four College Automated Photoelectric Telescope (FCAPT) have both improved periods, which are crucial to relating observations taken at different times, and better defined the shapes of their light curves (see, e.g. Adelman \& Brunhouse 1998). These results can be used to detect

Send offprint requests to: S.J. Adelman

* Tables 2, 3, 4 and 5 are only available in electronic form at the CDS via anonymous ftp to cdsarc.u-strasbg.fr (130.79.128.5) or via http://cdsweb.ustrasbg.fr/Abstract.html
Table 1. Photometric groups

\begin{tabular}{rllll}
\hline HD Number & Star Name & Type & $V$ & Spectral Type \\
\hline 90569 & 45 Leo & $v$ & 6.04 & A0pSiCr: \\
89774 & 42 Leo & $c$ & 6.12 & A1V \\
95216 & HR 4281 & $c h$ & 6.53 & F5V \\
& & & & \\
96707 & HR 4330 & $v$ & 6.06 & F0pSr \\
97138 & HR 4340 & $c$ & 6.40 & A3V \\
98772 & HR 4391 & $c h$ & 5.98 & A3V \\
& & & & \\
152308 & 49 Her & $v$ & 6.52 & B9.5pCr: \\
151862 & HR 6246 & $c$ & 5.91 & A1V \\
150483 & HR 6203 & $c h$ & 6.08 & A3Vn \\
& & & & \\
164429 & HR 6718 & $v$ & 6.48 & B9pSiSr \\
162132 & HR 6641 & $c$ & 6.43 & A2Vs \\
165358 & HR 6753 & $c h^{a}$ & 6.21 & A2V \\
166228 & HR 6792 & $c h^{a}$ & 6.32 & A2V \\
\hline
\end{tabular}

${ }^{a}$ HD 165358 was used as the check star for 1995-97 and HD 166228 for 1997-98.

changes in light curves between observing seasons, deduce information concerning the uniformity of the surface abundances, and study the period distribution of $\mathrm{mCP}$ stars. An important goal in this research is to derive abundances as a function of photospheric position and then use these abundances to test the theories of chemical differentiation. This permits investigators to learn about hydrodynamical processes at work in $\mathrm{mCP}$ (and other hot) star atmospheres.

This paper presents single-channel differential Strömgren photometry of four mCP stars obtained with the FCAPT, now at Washington Camp, AZ after being on Mt. Hopkins, AZ for six years (September 1990 - July 1996). For these stars Strömgren phtometry yields more astrophysical information than $U B V$ photometry. In part this is due to the $\lambda 5200$ broad, continuum feature's effect on $y$ values. For each photometric group (Table 1) of variable, comparison, and check stars, the telescope measures the dark count and then the sky-ch-c-v-c-v-c-v-c-ch-sky 
in each filter where sky, $c h, c$, and $v$ are respectively readings of the sky, the check star, the comparison star, and the variable star. The comparison and check stars were selected from supposedly non-variable stars near the variable on the sky that had similar $V$ magnitudes and $B-V$ colors using the Bright Star Catalogue (Hoffleit 1982) and the experience of other photometrists and checked later using photometry from the Hipparcos satellite (ESA 1997). Tables 2-5 contain the data and their yearly means and standard deviations. Corrections were not made for neutral density filter differences among the stars of each group. The standard deviations in Tables 2-5 of the check-comparison star differences indicate that these stars are constant for the period when they were observed.

We plot the $v$-c data for each variable star with the best published period to see if our data approximately confirm this period. Then we use the Scargle periodogram (Scargle 1982; Horne \& Baliunas 1986) and/or the clean algorithm (Roberts et al. 1987) with our data. Finally we adjust the period to make our data and any published data coincide as well as possible in phase. In Figs. 1-4 our values are indicated by plus signs, those of Winzer (1974) converted to our zero points as closed circles except for 49 Her where they are closed diamonds, and those of Burke \& Barr (1981) by closed diamonds for HR 6718 .

\section{45 Leo}

Provin (1953) found the sharp-lined star $(v \sin i=$ $10 \mathrm{~km} \mathrm{~s}^{-1}$, Abt \& Morrell 1995) 45 Leo (= HR 4101 $=\mathrm{HD} 90569=\mathrm{CX}$ Leo) to be photometrically constant, while Burke et al. (1970) thought it was probably variable. Winzer (1974) discovered it was a low amplitude photometric variable with a period of 1.4450 days, a result confirmed by Wolff \& Morrison (1975), but not Bonsack (1976). We are looking at a polar region. Leroy (1995) finds its polarization of $0.12 \%$ is due in part to interstellar polarization. It is not a star he expects to be usefully studied via broadband polarimetry.

We obtained 60 and 49 differential uvby observations of 45 Leo during the 1995-96 and 1996-97 observing seasons, respectively. As 45 Leo is not particularly variable longward of the Balmer jump, we studied the $u$ values and found that the most likely period was 1.4438 days. To bring the $U$ values of Winzer (1974) appropriately rezeroed into best agreement with our data resulted in a slight increase in the period. We also had to adjust the zero point of Winzer's ephemeris. Thus

$\operatorname{HJD}\left(u_{\max }\right)=2441460.954 \pm 0.002+(1.443820 \pm$ $0.000005) E$.

If Wolff \& Morrison (1975) had published their data in tabular form, we might have been able to reduce our errors. The amplitudes of variability are $0.042 \mathrm{mag}$ for $u$, $0.010 \mathrm{mag}$ for $b$, and $0.005 \mathrm{mag}$ for $v$ and $y$ (Fig. 1). Both $u$ and $b$ vary in phase in a single wave. The marginal variability of $v$ and $y$ are probably also in phase.

\section{HR 4330}

Winzer (1974) found that HR 4330 (= HD $96707=\mathrm{EP}$ $\mathrm{UMa})\left(v \sin i=33 \mathrm{~km} \mathrm{~s}^{-1}\right.$, Abt \& Morrell 1995) was a small amplitude light variable with a possible period of 0.8183 days. Its largest amplitude was in $V$. Burke \& Barr (1981) also observed this star, but one of their comparison stars was unfortunately variable. Matthews \& Wehlau (1985) did not find HR 4330 to be a rapid variable. According to Leroy (1995), it should show significant polarization, but does not. We obtained 101 sets of differential uvby observations of which 35 were from 1995-96, 37 from 1996-97, and 29 from 1997-98. Our periodograms indicated that the most likely period was near 3.516 days. We kept the zero point of Winzer's ephemeris and used

$$
\operatorname{HJD}\left(V_{\max }\right)=2441447.19+(3.5160 \pm 0.0001) E .
$$

The scatter seen in these light curves (Fig. 2) is greater than that for other mCP stars since these check and comparison stars are not as constant as those used with most mCP stars (Adelman et al. 1998). Hence HR 4330 should be reobserved with less variable check and comparison stars. Light maximum apparently comes near phase 0.9 rather than 0.0 as intended, but the shape of the maximum needs to be better defined. The amplitudes of HR 4330 are $0.02 \mathrm{mag}$ in $b$ and $0.025 \mathrm{mag}$ in $y$ with those for $u$ and $v$ probably smaller. The $b$ and $y$ curves are in phase. The $v$ values show scatter while those for $u$ suggest incipient structure.

\section{49 Her}

Winzer (1974) discovered that 49 Her (HR $6268=$ HD $152308=$ V823 Her) was a moderate amplitude photometric variable. He derived a period of 1.0978 days, but noted that the resonance period near 0.918 days was a possible alternative. Good quality differential uvby photometry was obtained with the FCAPT: 56 sets in 1996-97 and 19 sets in 1997-98. A periodogram analysis indicates that the period is close to 0.9366 days. For the best overlap with Winzer's $V$ data rezeroed to the FCAPT $y$ system, the period was lengthened to 0.93663 days. A slight adjustment in the zero epoch was also required. Thus

HJD(light maximum) $=2441453.925 \pm 0.003+$ $(0.93663 \pm 0.00002) E$.

The light curves (Fig. 3) show nearly flat bottomed minima and sharp well defined maxima. The curves for $u, v$, $b$, and $y$ are in phase. The minima appear to have two sub-minima with the one near phase 0.37 deeper than the one near phase 0.67. They are of order 0.005 mag fainter than the trend of adjacent values. The amplitudes are $0.055 \mathrm{mag}$ in $u, 0.028 \mathrm{mag}$ in $v, 0.035 \mathrm{mag}$ in $b$, 


\section{Leo}
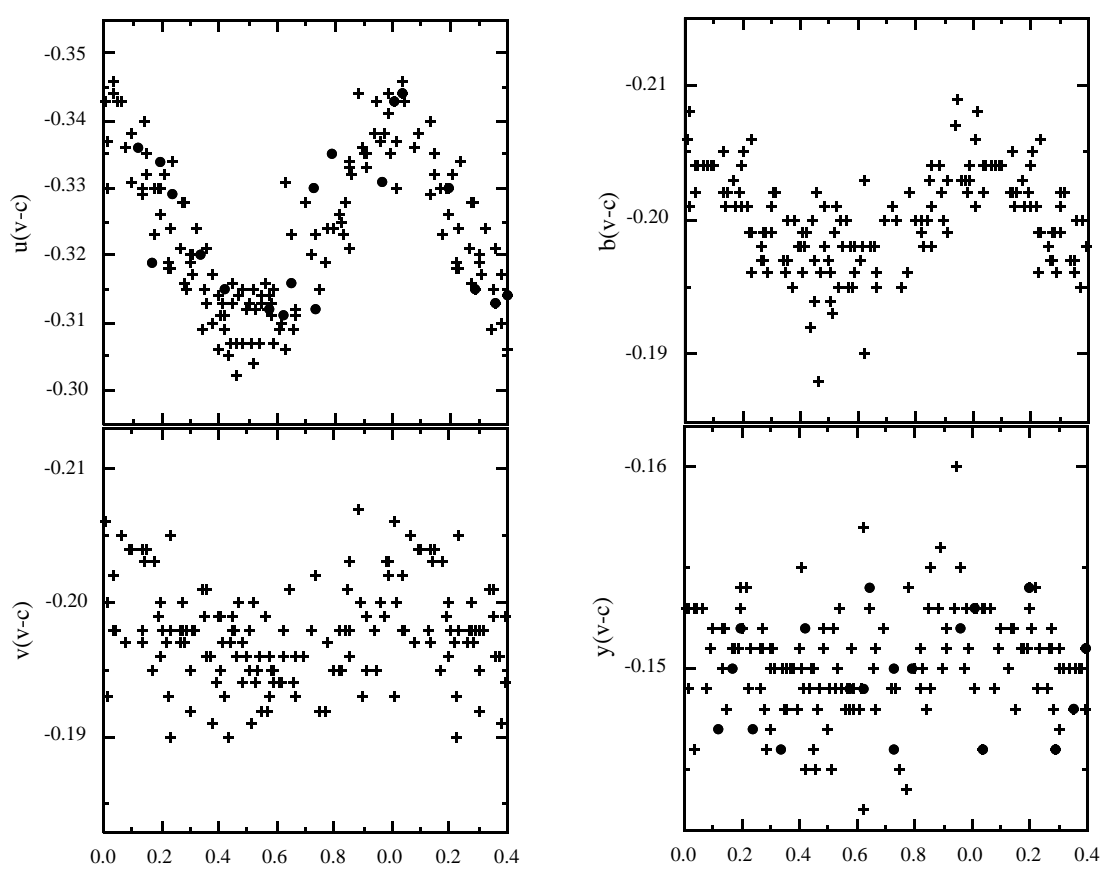

phase

Fig. 1. Photometry of 45 Leo plotted with the ephemeris $\operatorname{HJD}\left(u_{\max }\right)=2441460.954+1.443820 E$. The FCAPT uvby values are indicated by + signs while Winzer's $U$ and $V$ values as rezeroed to the $u$ and $y$ scales, respectively, are closed circles

\section{HR 4330}
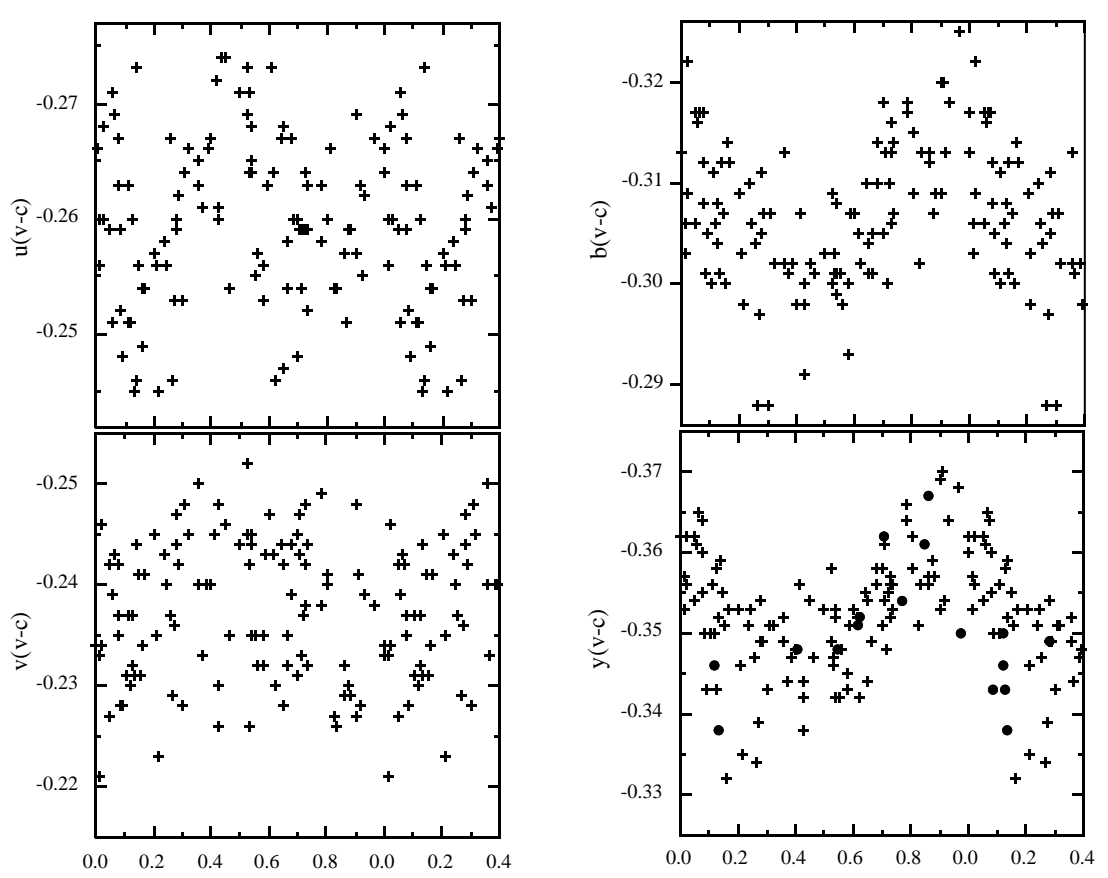

phase

Fig. 2. Photometry of HR 4330 plotted using the ephemeris $\operatorname{HJD}\left(y_{\max }\right)=2441447.19+3.5160$ E. The FCAPT uvby values are indicated by + signs while Winzer's $V$ values as rezeroed to the $y$ scale are closed circles 


\section{Her}
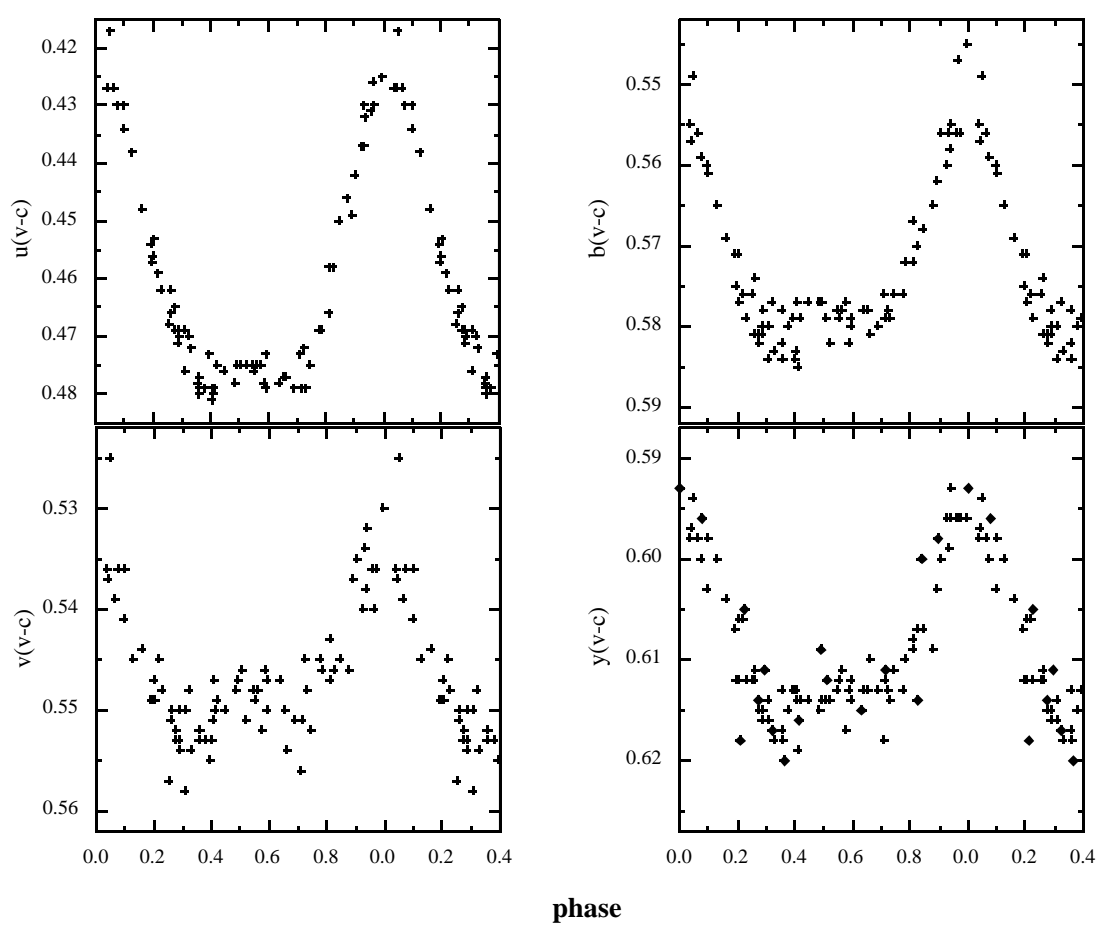

Fig. 3. Photometry of 49 Her plotted according to the ephemeris HJD(light maximum) $=2441453.925+0.93663$ E. The FCAPT $u v b y$ values are shown as + signs while Winzer's $V$ values as rezeroed to the $y$ scale are closed diamonds

\section{HR 6718}
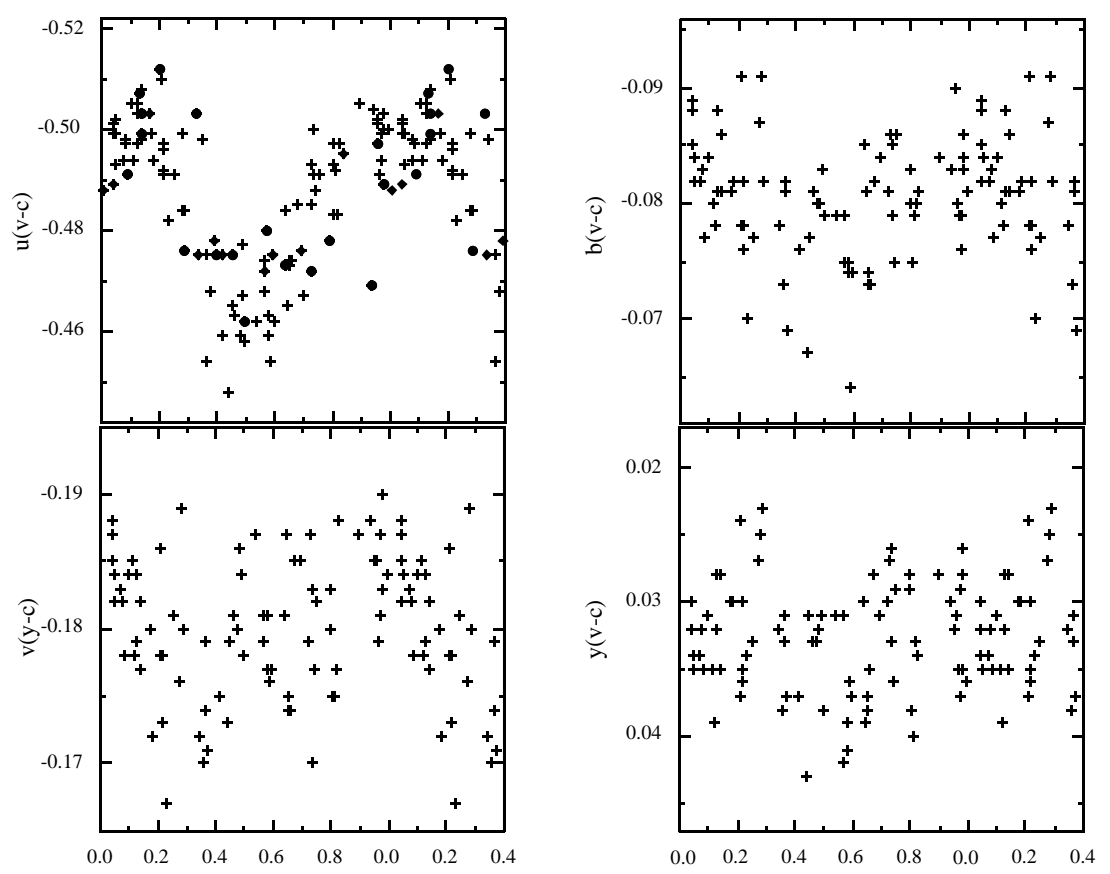

phase

Fig. 4. Photometry of HR 6718 plotted using the ephemeris $\operatorname{HJD}\left(u_{\max }\right)=2441450.808+0.51899 E$. The FCAPT uvby values are shown as + signs while Winzer's and Burke \& Barr's $U$ values as rezeroed to the $u$ scale are closed diamonds and closed circles, respectively 
and $0.023 \mathrm{mag}$ in $y$. That the second sub-minimum becomes less easy to see with increasing wavelength indicates that the flux distributions at the two sub-minima are not identical.

Abt \& Morrell (1995) find $v \sin i=95 \mathrm{~km} \mathrm{~s}^{-1}$. With a period of order one day, we must be looking at 49 Her with the rotation pole pointing about $20^{\circ}$ away from our line of sight. Thus we are seeing differences in the equatorial belt of $\pm 20^{\circ}$ latitude which are diminished by limb darkening. Light maximum may coincide with the transit of a magnetic pole across the observable stellar photosphere.

\section{HR 6718}

Winzer (1974) found that HR 6718 (= HD $164429=$ V771 Her) was a relatively low amplitude photometric variable with its largest amplitude of $0.03 \mathrm{mag}$ in $U$. The variation in $B$ is less than $0.01 \mathrm{mag}$ and is smaller than the $V$ amplitude. His period of 0.51747 days was confirmed by Catalano et al. (1979). Burke \& Barr (1981) obtained additional $U B V$ photometry, found slightly larger amplitudes, and essentially the same period of variability. For such a short period variable, its $v \sin i$ value of $85 \mathrm{~km} \mathrm{~s}^{-1}$ (Abt \& Morrell 1995) suggests it is seen very close to pole-on which in part explains its low degree of variability. Bohlender et al. (1993) made a measurement of its magnetic field: $640 \pm 480 \mathrm{G}$.

Seventy-seven sets of uvby differential values were obtained with the FCAPT, 11 in 1995-96, 35 in 1996-97, and 31 in 1997-98. A periodogram analysis of the $u$ data showed that the period was close to either a half-day or a day. When we used Winzer's (1974) and Burke \& Barr's (1981) $U$ data rezeroed to be as consistent as possible with our data we had increase the period slightly and make a slight adjustment to Winzer's zero epoch. Hence

$$
\begin{aligned}
& \operatorname{HJD}\left(u_{\max }\right)=2441450.808 \pm 0.005+(0.51899 \pm \\
& 0.00004) E .
\end{aligned}
$$

The uvby photometry plotted with this period (Fig. 4) shows that $u$ is definitely variable with an amplitude of 0.045 mag. Further $v, b$, and $y$ are probably variable with amplitudes of order $0.015 \mathrm{mag}, 0.01 \mathrm{mag}$, and $0.01 \mathrm{mag}$, respectively. In addition these light curves are probably shifted in phase by 0.1 with respect to $u$ which indicates a complicated surface distribution of abundances.

\section{Final comments}

This paper investigated the photometrically variability of four mCP stars studied by Winzer (1974). In most of the observed bandpasses, three of them are low amplitude variables and hence difficult objects to study. According to theory, the rotation of $\mathrm{mCP}$ stars is slowed by magnetic braking. Thus it is necessary to study $\mathrm{mCP}$ stars with the full range of observed amplitudes to determine the class period distribution. The moderate variable $49 \mathrm{Her}$ is a candidate the further study even though we are observing it close to pole-on. Babcock (1958) notes it is a spectrum variable, but its lines are too broad for effective measurements of the Zeeman effect via photography.

Acknowledgements. This work was supported in part by NSF grants AST-9115114 and 9528506. We appreciate the continuing efforts of L.J. Boyd, R.J. Dukes, Jr., and G.P. McCook to keep the FCAPT operating properly and also the useful comments of the referee Nancy Morrison.

\section{References}

Abt H.A., Morrell N.I., 1995, ApJS 99, 135

Adelman S.J., Brunhouse E.F., 1998, PASP 110, 1304

Adelman S.J., Pi C.-L.M., Rayle K.E., 1998, A\&AS 133, 197

Babcock H.W., 1958, ApJS 3, 141

Bohlender D.A., Landstreet J.D., Thompson I.B., 1993, A\&A 269, 355

Bonsack W.K., 1976, PASP 88, 19

Burke E.W. Jr., Barr T.H., 1981, PASP 93, 344

Burke E.W. Jr., Rolland W.W., Boy W.R., 1970, JRASC 64, 353

Catalano F.A., Maguzza A., Strazzulla G., 1979, IBVS 1695

ESA, 1997, The Hipparcos and Tycho Catalogs, SP-1200

Hoffleit D., 1982, The Bright Star Catalogue, 4th edition. Yale University Observatory, New Haven, CT

Horne J.H., Baliunas S.L., 1986, ApJ 302, 757

Leroy J.L., 1995, A\&AS 114, 79

Matthews J.M., Wehlau W.H., 1985, PASP 97, 841

Michaud G., Proffitt C.R., in ASP Conf. Proc. 44, Peculiar Versus Normal Phenomena in A-Type and Related Stars, Dworetsky M.M., Castelli F., Faraggiana R. (eds.). ASP, San Francisco, p. 439

Provin S.S., 1953, ApJ 118, 489

Roberts D.H., Lehar J., Dreher J.W., 1987, AJ 93, 968

Scargle J.D., 1982, ApJ 263, 835

Shore S.N.S.J., 1974, ApJ 161, 165

Winzer J.E., 1974, Ph. D. Dissertation, University of Toronto Wolff S.C., Morrison N.D., 1975, PASP 87, 231 